\title{
Frontières
}

\section{Les suicides}

\section{Extrait de l'article « Les suicides. L'Afrique et nous ", publié \\ dans la revue Frontières, vol. 1, n ${ }^{0}$ 2, automne 1988}

\section{Louis-Vincent Thomas}

Volume 21, numéro 1, automne 2008

Prévenir le suicide

URI : https://id.erudit.org/iderudit/037887ar

DOI : https://doi.org/10.7202/037887ar

Aller au sommaire du numéro

\section{Éditeur(s)}

Université du Québec à Montréal

ISSN

1180-3479 (imprimé)

1916-0976 (numérique)

Découvrir la revue

Citer ce compte rendu

Thomas, L.-V. (2008). Compte rendu de [Les suicides : extrait de l'article « Les suicides. L'Afrique et nous ", publié dans la revue Frontières, vol. $1, \mathrm{n}^{0} 2$, automne 1988]. Frontières, 21(1), 141-142. https://doi.org/10.7202/037887ar d'utilisation que vous pouvez consulter en ligne.

https://apropos.erudit.org/fr/usagers/politique-dutilisation/ 


\section{Extrait de l'article «Les suicides. L'Afrique et nous», publié dans la revue Frontières, vol. 1, n² 2, automne 1988}

\section{Louis-Vincent Thomas (1922-1994), professeur de sociologie et d'anthropologie, Sorbonne Paris V (1968-1988).}

De façon générale, le suicide qualifie l'acte de se tuer (ou de tenter de le faire), de se faire tuer, ou seulement de se laisser mourir en vertu d'une décision personnelle préalable, enfin de demander qu'on mette fin à nos jours (suicide assisté). On ne saurait, en effet, parler de suicide qu'au pluriel, surtout si l'on s'attache aux conditions, au sens, aux causes et aux finalités de ces actes mortifères et de certains de leurs équivalents.

Le suicide, par exemple, recouvre diverses attitudes et motivations que l'on peut ramener à quatre (Beachler, 1975) : 1) les réactions de fuite à une déchéance, à une trop grande souffrance, à un échec ou une situation inacceptable, à une responsabilité excessive, à la perte du contrôle de soi ou de l'environnement (suicide escapiste) ; 2) les attitudes d'agression à l'endroit d'un monde qui nous abandonne, ne répond pas à nos attentes ou nous semble absurde (suicide agressif); 3) les comportements d'oblation tels que se sacrifier à un code d'honneur, se détruire pour que meure l'ennemi (kamikaze), refuser de survivre au chef aimé, donner sa vie pour Dieu (martyre désiré), ou pour défendre une cause politique... Tout cela peut impliquer une sorte d'union mystique avec la mort (suicide oblatif) ; 4) les conduites ludiques et ordaliques automortifères : le jeu, notoirement le jeu de hasard, s'apparente au geste du suicidant. En effet:

Tous les grands joueurs sont hantés par la mort, prendre un risque c'est défier la mort, et tout gain est considéré comme un surplus de vie. Mais paradoxalement, la psychologie des joueurs montre que c'est le désir de mort qui pousse le joueur. On sait bien, le calcul des probabilités est

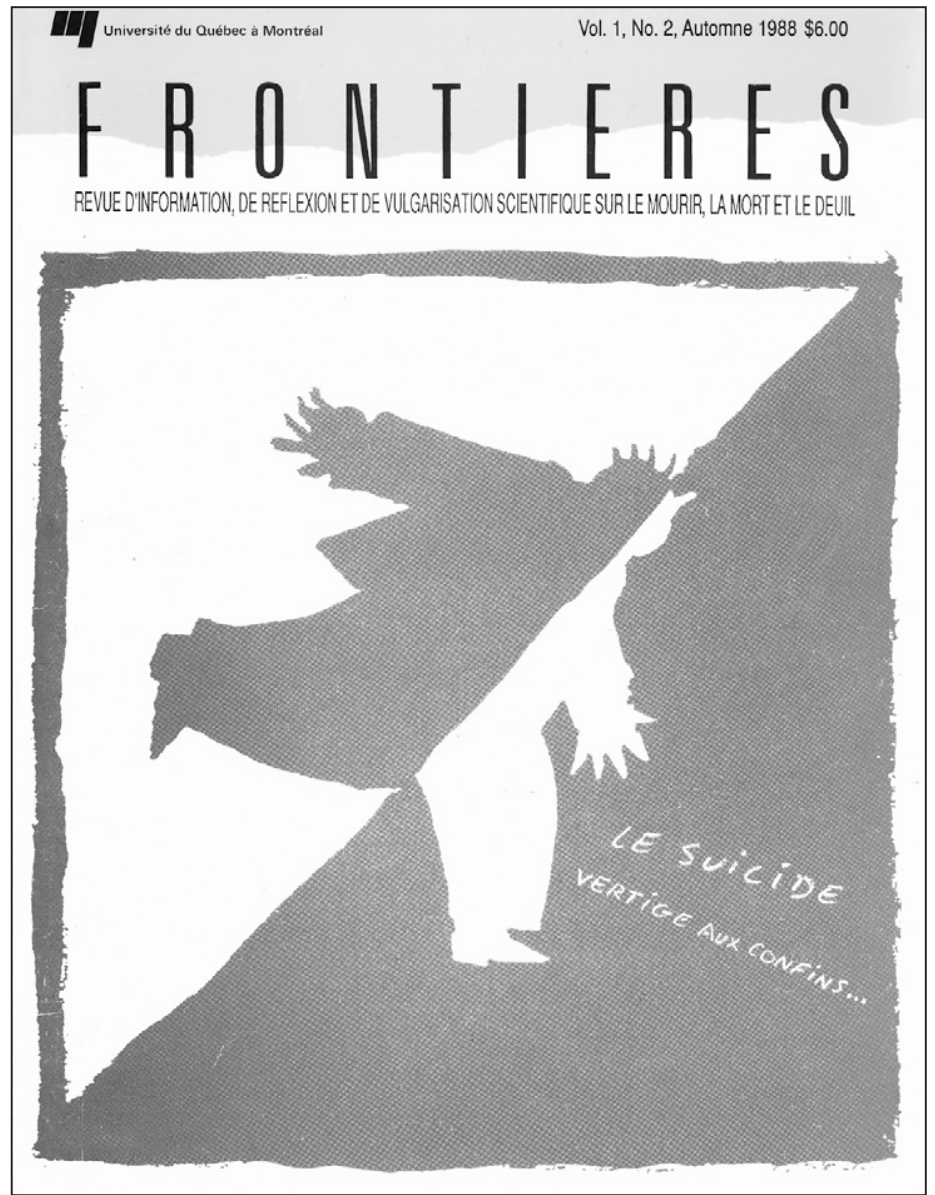


péremptoire à ce sujet, que le joueur doit perdre. C'est cela qui rend le jeu excitant, il n'est qu'illusion de triomphe, mais c'est cela aussi qui donne cette saveur inestimable au gain, signe du destin (Menahem, 1973).

Le goût du risque, dans la course automobile, l'escalade en montagne, la corrida, les traversées en solitaire... ont un goût rentré du suicide; tout comme le fait de jouer à la roulette russe. Autant d'attitudes ambivalentes où l'on veut à la fois vivre et mourir; où vivre jusqu'aux limites de l'impossible implique le risque, désiré ou imposé, de mourir. J.M. Brohm (1986; 1987) l'a fort habilement montré à propos de certaines conduites sportives.

$[\ldots]$

\section{LE SUICIDE ET L'OCCIDENT AUJOURD'HUI}

Diverses situations propres au monde occidental d'aujourd'hui ont à voir avec le suicide.

- L'obsession de l'apocalypse s'avère ambivalente car celle-ci pourrait bien prendre la forme d'un holocauste généralisé inconsciemment consenti. Certaines enquêtes que nous avons menées voient dans la mort atomique une explosion érotique, à valence sexuelle indiscutable (la première bombe s'est prénommée Gilda, évoquant une star célèbre). On y parle de lumière, de milliers de soleils, d'éblouissement. L'accompagnement obligé en est la chaleur, «énergie pure, plus noble encore que la vie», dit une jeune fille. Enfin la déchirure, l'éclatement, la dislocation sont des termes qui appartiennent aussi au paroxysme sexuel. Ce qui nous renvoie encore aux mythes archaïques du retour au feu primordial et de la renaissance pan-cosmique.

- Sur un point plus concret, n'oublions pas que les suicides ratés, fréquents chez les jeunes (ils ne voulaient pas vraiment mourir) deviennent très rares chez les vieillards: cette fois le suicide n'est pas un appel, un chantage, ou une vengeance, mais exprime un réel désir d'en finir avec une société qui les rejette, les oublie ou les exploite (politiquement, économiquement). Cela pose, bien entendu, à nos sociétés qui vieillissent (nombre accru de vieux; vieillissement du vieillissement) des problèmes difficiles à résoudre: que faire pour que les personnes âgées vivent dignement et le plus confortablement possible ce qui leur reste à vivre ? Le temps nous manque pour aborder comme il se doit une question aussi difficile et aussi urgente; ce que l'on peut avancer avec certitude, c'est que dans les hôpitaux de longs séjours les demandes d'euthanasie ou de suicide assisté se font rares si les vieux sont accompagnés médicalement, socialement et affectivement, voire spirituellement.

- Enfin notre monde a généré des groupes de pression qui militent avec conviction en faveur du droit à la mort, donc au suicide. Ne faudrait-il pas, à ce propos, écrire avec A.M. Piper qu'on ne peut ni entendre, ni proscrire le suicide. La vie est une condition nécessaire, mais non suffisante pour que l'homme existe en tant qu'homme.

La condition suffisante pour que l'homme existe en tant qu'homme, c'est-à-dire d'une manière digne de l'homme, c'est la liberté. Ce n'est qu'en tant qu'être vivant libre que l'homme est totalement homme. Une action qui n'est moralement ni ordonnée ni défendue reçoit, en tant que posée librement, son statut intermédiaire d'action permise, du fait qu'elle ne viole pas le principe de la liberté, comme les actions défendues, mais ne le confirme pas non plus comme les actions ordonnées qui ouvrent la liberté en un sens originel. Le suicide (non pathologique, bien réfléchi) est cependant un acte accompli en faveur de la liberté ; cet acte n'ouvre sans doute pas une nouvelle liberté pour celui qui agit ainsi, mais achève pour lui une fois pour toutes cette forme de réalisation de l'être-homme. Pourtant il fait en même temps apercevoir que son auteur ne veut pas savoir abolir la validité du principe de la liberté, mais, d'une façon exactement inverse, qu'il reconnaît ce principe et, parce qu'il le reconnaît, préfère la mort à la vie non libre et par là à une vie qui n'est plus digne de l'homme, qui ne vaut plus la peine d'être vécue. Une vie qui ne peut être vécue qu'au prix de l'humanité est moins, elle est plus indigne [...] que le simple fait de ne pas vivre (Pieper, 1985, p. 70-71).

Nous sommes en mesure de conclure.

- Le suicide pose avant tout la question du sens : sens de la vie, sens du geste meurtrier, quête du sens et refus du non-sens.

- Aussi est-il un langage qu'il faut interpréter, dont on ignore le plus souvent le code et qui reste presque toujours un raté de la communication.

- Le suicidant n'est ni vraiment coupable ni vraiment malade (sauf cas limites), pas plus que son geste exprime tout à fait la liberté et entièrement l'impérieuse nécessité. Ni toutepuissance, ni totale impuissance. Ce serait trop simple.

- Le suicide nous introduit dans la plus grande des complexités et la plus parfaite des diversités rendant ainsi vaine toute possibilité de jugement. Si tout doit être fait impérativement pour aider le suicidaire (psychologiquement, socialement, médicalement, surtout s'il est solitaire), en revanche, eu égard à l'ambivalence que suppose cet acte, on ne doit ni l'interdire ni l'exiger. Au nom même de la dignité et la liberté humaine. $[\ldots]$

\section{Bibliographie}

BEACHLER, J. (1975). Les suicides, Paris, Calmann-Levy.

BROHM, J.M. (1986). «Du sport suicidaire au suicide sportif », dans Quel corps, Montreuil, Éditions de la Passion.

BROHM, J.M. (1987). «Urgences sportives ; l'extrême limite », Actions et recherches sociales, $\mathrm{n}^{\circ} 2$.

MENAHEM, R. (1973). La mort apprivoisée, Paris, Éditions Universitaires.

PIEPER, A.M. (1985). "Arguments éthiques pour la permission du suicide », Concilium, Suicide et droit à la mort, no 199, p. 63-74. 